Bull. Soc. math. France

130 (2), 2002, p. 233-251

\title{
OPÉRADES DIFFÉRENTIELLES GRADUÉES SUR LES SIMPLEXES ET LES PERMUTOÈDRES
}

\author{
PAR FRÉDÉRIC CHAPOTON
}

\begin{abstract}
RÉSumÉ. - On définit plusieurs opérades différentielles graduées, dont certaines en relation avec des familles de polytopes : les simplexes et les permutoèdres. On obtient également une présentation de l'opérade $K$ liée aux associaèdres introduite dans un article antérieur.

ABstract (Differential graded operads related to simplices and permutohedra)

We define several differential graded operads, some of them being related to families of polytopes : simplices and permutohedra. We also obtain a presentation by generators and relations of the operad $K$ on associahedra introduced in a previous article.
\end{abstract}

\section{Introduction}

Les algèbres associatives commutatives, les algèbres associatives et les algèbres de Lie font partie du paysage mathématique depuis longtemps.

Plus récemment sont apparues les algèbres de Leibniz, les digèbres, les algèbres dendriformes et les algèbres zinbiel, toutes introduites par Loday avec des motivations en K-théorie, voir [5], [6], [7], [8].

Texte reçu le 28 février 2001, révisé le 18 octobre 2001, accepté le 9 novembre 2001 Frédéric Chapoton, LACIM, Université du Québec à Montréal, CP 8888 succ. centre ville, Montréal Québec H3C 3P8, (Canada) - E-mail : chapoton@lacim.uqam.ca Classification mathématique par sujets (2000). - 18D50, 52B11, 17A32, 17D25.

Mots clefs. - Opérades, permutoèdres, simplexes, associaèdres, algèbres pré-Lie, algèbres dendriformes, algèbres de Leibniz. 
Ces types d'algèbres s'organisent en un diagramme d'opérades, qui se complète par les algèbres permutatives et les algèbres pré-Lie ${ }^{(1)}$, voir [1], [3].

Par ailleurs, la relation entre l'opérade des algèbres dendriformes et les sommets des polytopes de Stasheff mène à la définition d'une opérade différentielle graduée $K$ sur toutes les faces des polytopes de Stasheff [2]. La place que prend cette opérade parmi les précédentes incite à compléter encore le diagramme, ce que nous faisons ici.

On va définir les seconde et quatrième lignes du diagramme commutatif suivant, où les flèches marquées q.i. sont des quasi-isomorphismes.

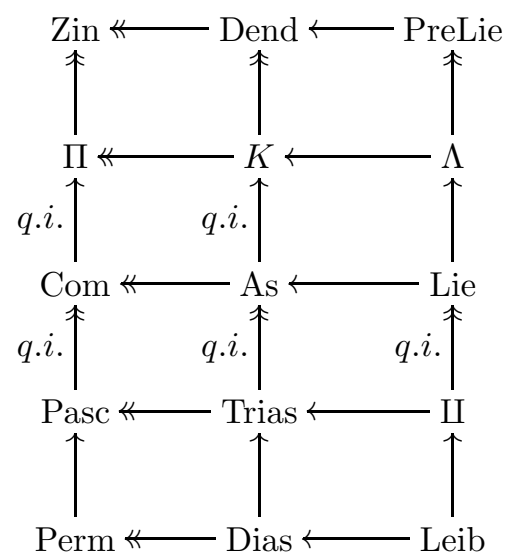

La symétrie centrale de ce diagramme correspond à la dualité quadratique des opérades quadratiques binaires.

L'opérade $K$ a été définie dans la première partie de [2] ; elle est en relation avec les associaèdres. Ici, on commence par définir deux opérades $\Pi$ (faisant intervenir les permutoèdres) et Pasc (liée aux simplexes). On donne ensuite une présentation par générateurs et relations de ces opérades, qui montre en particulier que ces trois opérades sont quadratiques binaires. Les résultats de Markl [10] sur les lois distributives permettent de démontrer que $\Pi$ est de Koszul.

On donne ensuite une présentation des opérades $\amalg, \Lambda$ et Trias, duales quadratiques des précédentes au sens de Ginzburg et Kapranov [4]. L'opérade Trias provient d'une opérade non-symétrique Trias'. On démontre que Trias' est isomorphe à l'opérade Pasc vue comme opérade non-symétrique.

Durant la préparation de cet article, j'ai reçu une note de Loday et Ronco [9] annonçant des résultats similaires pour des variantes non-graduées des opérades $K$ et Trias.

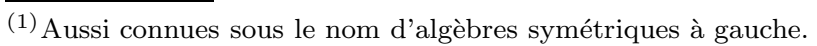

TOME $130-2002-\mathrm{N}^{\mathrm{O}} 2$ 


\section{Opérade $\Pi$}

On se place dans toute la suite de l'article dans la catégorie monoïdale symétrique des complexes de $\mathbb{Z}$-modules.

On définit une opérade différentielle graduée П. Les complexes sous-jacents sont les complexes de cochaînes cellulaires des permutoèdres.

1.1. Complexes sous-jacents. - Soit $I$ un ensemble fini. On note $\Pi(I)$ le $\mathbb{Z}$-module libre engendré par les expressions de la forme $\pi_{1} \otimes \pi_{2} \otimes \cdots \otimes \pi_{p}$, où $\pi_{j}=i_{j, 1} \wedge \cdots \wedge i_{j, p_{j}}$ avec $i_{j, k} \in I$ et où chaque élément de $I$ apparaît dans un certain $\pi_{j}$ et un seul, modulo les relations d'antisymétrie des produits extérieurs. Ces expressions correspondent à des partitions ordonnées de $I$, en bijection avec les faces du permutoèdre correspondant à $I$.

Si $\pi_{j}=i_{j, 1} \wedge \cdots \wedge i_{j, p_{j}}$, on appelle dimension de $\pi_{j}$, l'entier $p_{j}-1$, noté $\operatorname{dim}\left(\pi_{j}\right)$. On munit $\Pi(I)$ de la graduation par la dimension en posant, si $\pi=\pi_{1} \otimes \pi_{2} \otimes \cdots \otimes \pi_{p}$

$$
\operatorname{dim}(\pi)=\sum_{j=1}^{p} \operatorname{dim}\left(\pi_{j}\right)
$$

et d'une différentielle $d$ de degré +1 définie par la formule suivante :

$$
d(\pi)=\sum_{j=1}^{p-1}(-1)^{\operatorname{dim}\left(\pi_{1}\right)+\cdots+\operatorname{dim}\left(\pi_{j}\right)} \pi_{1} \otimes \cdots \otimes \pi_{j} \wedge \pi_{j+1} \otimes \cdots \otimes \pi_{p} .
$$

On vérifie sans difficulté que $d$ est de carré nul.

1.2. Composition de l'opérade. - Soient $I$ et $J$ deux ensembles finis et $i \in I$. Soient $\pi=\pi_{1} \otimes \pi_{2} \otimes \cdots \otimes \pi_{p} \in \Pi(I)$ et $\mu=\mu_{1} \otimes \mu_{2} \otimes \cdots \otimes \mu_{q} \in \Pi(J)$. On va définir la composition $\pi \circ_{i} \mu$, appartenant à $\Pi(I \backslash\{i\} \sqcup J)$. Soit $\ell$ l'unique indice tel que $i$ apparaît dans $\pi_{\ell}$. Quitte à changer de signe, on peut supposer que $\pi_{\ell}=\pi_{\ell}^{\prime} \wedge i$, avec la convention que, si $\pi_{\ell}=i$, alors $\pi_{\ell}^{\prime} \wedge \nu=\nu$ pour tout $\nu$.

On pose alors

(3) $\pi \circ_{i} \mu=\sum_{\sigma} \varepsilon(\sigma) \pi_{1} \otimes \cdots \otimes \pi_{\ell-1} \otimes \pi_{\ell}^{\prime} \wedge \mu_{1} \otimes \sigma\left(\pi_{\ell+1} \otimes \cdots \otimes \pi_{p}, \mu_{2} \otimes \cdots \otimes \mu_{q}\right)$,

où $\sigma$ décrit les battages de $\pi_{\ell+1}, \ldots, \pi_{p}$ avec $\mu_{2}, \ldots, \mu_{q}, \sigma(\cdots)$ est le produit tensoriel de $\pi_{\ell+1}, \ldots, \pi_{p}, \mu_{2}, \ldots, \mu_{q}$ dans l'ordre spécifié par $\sigma$ et $\epsilon(\sigma)$ désigne le signe obtenu par la règle de Koszul (en utilisant la volte graduée par la dimension) pour passer de $\pi_{\ell+1} \otimes \cdots \otimes \pi_{p} \otimes \mu_{1} \otimes \cdots \otimes \mu_{q}$ à $\mu_{1} \otimes \sigma\left(\pi_{\ell+1} \otimes\right.$ $\left.\cdots \otimes \pi_{p}, \mu_{2} \otimes \cdots \otimes \mu_{q}\right)$.

Proposition 1. - П est une opérade différentielle graduée. 
Démonstration. — L'élément 1 de $\Pi(\{1\})$ est une unité. La preuve que la composition est un morphisme de complexes est un calcul long mais sans difficulté, de même que celle de l'associativité. L'équivariance, i.e. la fonctorialité de la définition sur le groupoïde des ensembles finis, est immédiate.

\subsection{Présentation par générateurs et relations}

Proposition 2. - L'opérade $\Pi$ est engendrée par $\Pi(\{1,2\})$.

Démonstration. - On montre d'abord par récurrence que $1 \wedge 2 \wedge \cdots \wedge n$ s'obtient par composition itérée de $1 \wedge 2$ en utilisant la relation

$$
(1 \wedge 2 \wedge \cdots \wedge n-1 \wedge \star) o_{\star}(n \wedge n+1)=(1 \wedge 2 \wedge \cdots \wedge n+1) .
$$

On montre de même que $1 \otimes 2 \otimes \cdots \otimes n$ s'obtient par composition itérée de $1 \otimes 2$ en utilisant la relation

(5) $\quad(1 \otimes 2 \otimes \cdots \otimes n-1 \otimes \star) \circ_{\star}(n \otimes n+1)=(1 \otimes 2 \otimes \cdots \otimes n+1)$.

En effectuant successivement la composition d'un élément de la forme $i_{1} \wedge i_{2} \wedge \cdots \wedge i_{p}$ dans chaque terme d'un élément de la forme $1 \otimes 2 \otimes \cdots \otimes q$, on obtient le résultat voulu.

On a dans $\Pi$ les relations suivantes :

$$
\begin{aligned}
& (1 \otimes 2) \circ_{1}(1 \otimes 2)=(1 \otimes 2) \circ_{2}(1 \otimes 2)+(1 \otimes 2) \circ_{2}(2 \otimes 1), \\
& (1 \wedge 2) \circ_{2}(1 \otimes 2)=(1 \otimes 2) \circ_{1}(1 \wedge 2)=\tau\left((1 \wedge 2) \circ_{1}(2 \otimes 1)\right), \\
& (2 \wedge 1) \circ_{1}(1 \wedge 2)=(1 \wedge 2) \circ_{2}(1 \wedge 2),
\end{aligned}
$$

où $\tau$ est le cycle $3 \rightarrow 2 \rightarrow 1 \rightarrow 3$ agissant dans $\Pi(\{1,2,3\})$.

Proposition 3. - L'opérade $\Pi$ est isomorphe au quotient de l'opérade libre sur $\Pi(\{1,2\})$ par l'idéal engendré par les relations ci-dessus. En particulier $\Pi$ est une opérade quadratique binaire.

Démonstration. - Soit $\mathcal{F}$ l'opérade libre sur $\Pi(\{1,2\})$, Rel les relations cidessus et Quot l'opérade quotient de $\mathcal{F}$ par l'idéal engendré par Rel. On a un morphisme d'opérades de Quot $\rightarrow \Pi$, surjectif par la proposition 2 .

On remarque que la relation (6) est celle de l'opérade Zin et que la relation (8) est celle de la suspension de l'opérade Com. Par ailleurs, on vérifie que la relation (7) définit une loi distributive au sens de Markl [10] reliant l'opérade Zin et la suspension de Com. Comme les $\mathbb{Z}$-modules sous-jacents à Zin et Com sont libres, les $\mathbb{Z}$-modules sous-jacents à Quot sont également libres.

Pour montrer que la projection Quot $\rightarrow \Pi$ est un isomorphisme, il suffit de montrer l'égalité des rangs des $\mathbb{Z}$-modules libres sous-jacents. On rappelle la définition de la série génératrice d'une opérade différentielle graduée $\mathcal{P}$ :

$$
g_{\mathcal{P}}(x, t)=\sum_{n \geq 1} \sum_{k} \operatorname{dim} \mathcal{P}^{k}(n)(-t)^{k} \frac{x^{n}}{n !} .
$$

TOME $130-2002-\mathrm{N}^{\mathrm{O}} 2$ 
Par une propriété des lois distributives, la série génératrice de Quot s'obtient par composition de celles de Zin et de la suspension de Com. On vérifie qu'elle coïncide avec celle de $\Pi$, voir le tableau récapitulatif à la fin de l'article.

La différentielle de $\Pi$ est donc uniquement déterminée par le respect de la structure d'opérade et les conditions suivantes :

$$
\begin{aligned}
& d(1 \otimes 2)=1 \wedge 2, \\
& d(1 \wedge 2)=0 .
\end{aligned}
$$

On en déduit la description suivante des П-algèbres.

DÉFInItion 1. - Une П-algèbre est la donnée d'un complexe de cochaînes $V$ et d'applications $\prec: V \otimes V \rightarrow V$ et $\times: V \otimes V \rightarrow V[-1]$ telles que pour tous $x, y, z$ dans $V$,

$$
\begin{aligned}
(x \prec y) \prec z & =x \prec(y \prec z)+(-1)^{y z} x \prec(z \prec y), \\
x \times(y \prec z) & =(x \times y) \prec z=(-1)^{y z+z}(x \prec z) \times y, \\
x \times y & =(-1)^{x y+x+y+1} y \times x, \\
(x \times y) \times z & =x \times(y \times z)
\end{aligned}
$$

et

$$
\begin{array}{r}
(d x \prec y)+(-1)^{x}(x \prec d y)-d(x \prec y)=(-1)^{x} x \times y, \\
(d x \times y)+(-1)^{x+1}(x \times d y)-d(x \times y)=0 .
\end{array}
$$

Remarque 1. - On utilise ici et plus loin l'abus de notation qui consiste, dans les exposants de $(-1)$, à écrire $x, y, z$ pour $\operatorname{dim}(x), \operatorname{dim}(y), \operatorname{dim}(z)$.

Proposition 4. - L'opérade П est de Koszul.

Démonstration. - Soit $\Pi^{\prime}$ l'opérade obtenue en munissant $\Pi$ de la différentielle nulle. Les opérades Com et Zin sont de Koszul et $\Pi^{\prime}$ est décrite par une loi distributive, donc $\Pi^{\prime}$ est de Koszul par le théorème 4.5 de [10]. Par ailleurs, la koszulité d'une opérade différentielle dont les complexes sous-jacents sont bornés est une conséquence de la koszulité de la même opérade avec la différentielle nulle. En effet, les complexes Bar augmentés sont alors acycliques pour la différentielle totale car munis d'une filtration finie à quotients acycliques. Ceci entraîne que $\Pi$ est de Koszul.

On observe, par ailleurs, que le quotient de $\Pi$ par l'idéal différentiel formé des éléments de dimension non nulle est une opérade (de différentielle nulle) isomorphe à l'opérade Zin des algèbres zinbiel.

D'autre part, on a un morphisme de Com muni de la différentielle nulle dans $\Pi$. C'est un quasi-isomorphisme car les complexes sous-jacents sont les 
complexes cellulaires des permutoèdres, de sorte que l'homologie est en degré 0 avec action triviale des groupes symétriques.

\section{Opérade Pasc}

On définit une opérade différentielle graduée Pasc. Les complexes sousjacents sont les complexes de chaînes des simplexes.

2.1. Complexes sous-jacents. - Si $I$ est un ensemble fini non vide, on note $\mathbb{Z}^{I}$ le $\mathbb{Z}$-module libre de base $\left(e_{i}^{I}\right)_{i \in I}$. Soit $\operatorname{Ex}(I)$ l'algèbre extérieure avec unité sur $\mathbb{Z}^{I}$, munie de la graduation standard.

Soit $i \in I$; on note $\theta_{i}^{I}$ la dérivation (à gauche) de degré -1 de $\operatorname{Ex}(I)$ définie sur les générateurs par

$$
\theta_{i}^{I}\left(e_{j}^{I}\right)=\delta_{i j} .
$$

On munit $\operatorname{Ex}(I)$ de la différentielle $d$ de degré -1 définie par la formule

$$
d(x)=\sum_{i \in I} \theta_{i}^{I}(x) .
$$

On définit $\operatorname{Pasc}(I)$ comme le complexe quotient de $\operatorname{Ex}(I)$ par l'unité de $\operatorname{Ex}(I)$, muni d'une graduation décalée nommée dimension : si $P$ est une partie non vide de $I$, on pose

$$
\operatorname{dim}\left( \pm \wedge_{i \in P} e_{i}^{I}\right)=\operatorname{Card}(P)-1 .
$$

On note encore $\theta_{i}^{I}$ l'application obtenue par passage au quotient. Elle vérifie

$$
\theta_{i}^{I}(x \wedge y)=\theta_{i}^{I}(x) \wedge y+(-1)^{1+\operatorname{dim}(x)} x \wedge \theta_{i}^{I}(y),
$$

pour $x, y$ homogènes de dimensions non nulles dans $\operatorname{Pasc}(I)$.

2.2. Composition. - Soient $I, J$ deux ensembles finis, $P \subset I$ et $Q \subset J$ des parties non vides et $i \in I$. On choisit une énumération arbitraire de $P$ et $Q$ : $P=\left\{i_{1}, \ldots, i_{p}\right\}$ et $Q=\left\{j_{1}, \ldots, j_{q}\right\}$.

Soient $x=e_{i_{1}}^{I} \wedge \cdots \wedge e_{i_{p}}^{I}$ et $y=e_{j_{1}}^{J} \wedge \cdots \wedge e_{j_{q}}^{J}$. On définit la composition $x \circ_{i} y$, appartenant à $\operatorname{Pasc}(I \backslash\{i\} \sqcup J)$, par

$$
x \circ_{i} y=\left\{\begin{array}{l}
(-1)^{\operatorname{dim}(x)} \theta_{i}^{I \sqcup J}(x \wedge y) \text { si } i \in P, \\
x \text { si } i \notin P \text { et } \operatorname{dim} y=0, \\
0 \text { si } i \notin P \text { et } \operatorname{dim} y>0 .
\end{array}\right.
$$

Remarque 2. - Dans cette définition, les mêmes produits extérieurs sont implicitement considérés dans des algèbres extérieures différentes, via les identifications canoniques.

Proposition 5. - Pasc est une opérade différentielle graduée.

TOME $130-2002-\mathrm{N}^{\mathrm{O}} 2$ 
Démonstration. - L'élément $e_{1}^{\{1\}}$ de $\operatorname{Pasc}(\{1\})$ est clairement une unité. La preuve que la composition est un morphisme de complexes passe par une étude des différents cas possibles. De même, on montre l'associativité et l'équivariance de la composition en distinguant les différentes situations.

\subsection{Présentation par générateurs et relations}

Proposition 6. - L'opérade Pasc est engendrée par $\operatorname{Pasc}(\{1,2\})$.

Démonstration. - On montre d'abord par récurrence que, pour tout ensemble $I$ et tout $i \in I$, on peut obtenir $e_{i}^{I}$. On utilise la relation

$$
e_{i}^{I \backslash\{j, k\} \sqcup\{\star\}} \circ_{\star} e_{j}^{\{j, k\}}=e_{i}^{I},
$$

pour $i, j, k \in I$ deux à deux distincts.

On montre de même que, pour tout ensemble $I$, on peut obtenir $\wedge_{i \in I} e_{i}^{I}$. On utilise la formule

$$
\left(\wedge_{i \in I \backslash\{j, k\}} e_{i}^{I \backslash\{j, k\} \sqcup\{\star\}} \wedge e_{\{\star\}}^{I \backslash\{j, k\} \sqcup\{\star\}}\right) \circ_{\star}\left(e_{j}^{\{j, k\}} \wedge e_{k}^{\{j, k\}}\right)=\wedge_{i \in I} e_{i}^{I},
$$

pour $i, j, k \in I$ deux à deux distincts.

Enfin, on déduit le résultat voulu des deux points précédents en utilisant la relation

$$
e_{\star}^{I \backslash P \sqcup\{\star\}} \circ_{\star}\left(\wedge_{i \in P} e_{i}^{P}\right)=\wedge_{i \in P} e_{i}^{I},
$$

pour $P$ une partie non vide de $I$.

En notant $e_{i}=e_{i}^{\{1,2\}}$ pour $i=1$ ou 2 , on a dans Pasc les relations suivantes :

$$
\begin{aligned}
e_{1} \circ_{1} e_{1} & =\tau^{2}\left(e_{2} \circ_{1} e_{1}\right), \\
e_{2} \circ_{1} e_{1} & =e_{2} \circ_{1} e_{2}, \\
e_{1} \circ_{1} e_{2} & =\tau\left(e_{2} \circ_{1} e_{2}\right), \\
e_{2} \circ_{1}\left(e_{1} \wedge e_{2}\right) & =0 \\
\tau\left(\left(e_{1} \wedge e_{2}\right) \circ_{1} e_{2}\right) & =e_{1} \circ_{1}\left(e_{1} \wedge e_{2}\right), \\
-\tau^{2}\left(\left(e_{1} \wedge e_{2}\right) \circ_{1} e_{1}\right) & =e_{1} \circ_{1}\left(e_{1} \wedge e_{2}\right), \\
\left(e_{1} \wedge e_{2}\right) \circ_{1}\left(e_{1} \wedge e_{2}\right) & =\tau\left(\left(e_{1} \wedge e_{2}\right) \circ_{1}\left(e_{1} \wedge e_{2}\right)\right),
\end{aligned}
$$

où $\tau$ est le cycle $3 \rightarrow 2 \rightarrow 1 \rightarrow 3$.

Proposition 7. - L'opérade Pasc est isomorphe au quotient de l'opérade libre sur $\operatorname{Pasc}(\{1,2\})$ par l'idéal engendré par les relations ci-dessus. En particulier Pasc est une opérade quadratique binaire. 
Démonstration. - Soit $\mathcal{F}$ l'opérade libre $\operatorname{sur} \operatorname{Pasc}(\{1,2\})$, Rel les relations cidessus et Quot l'opérade quotient de $\mathcal{F}$ par l'idéal engendré par Rel. Par la proposition 6, on a un morphisme surjectif d'opérades $\phi$ de Quot $\rightarrow$ Pasc. La démonstration consiste à construire un inverse $\psi$ par récurrence. On peut négliger les questions de signes et donc raisonner sur des parties plutôt que sur des produits extérieurs. On dit que la composition $P \circ_{i} Q$ est interne si $i \in P$ et externe sinon.

Supposons donc un inverse $\psi$ construit jusqu'au rang $n-1$. Soit $I$ un ensemble à $n \geq 3$ éléments et $P$ une partie non vide de $I$. On distingue deux cas : ou bien $P$ contient au moins deux éléments, ou bien son complémentaire contient au moins deux éléments. Dans le premier cas, on peut écrire $P$ comme composé interne de la partie $p=\{1,2\}$ de $\{1,2\}$ dans une partie $P^{\prime}$ d'un ensemble de cardinal $n-1$. Dans le second cas, on peut écrire $P$ comme composé externe de la partie $p=\{1\}$ de $\{1,2\}$ dans une partie $P^{\prime}$ d'un ensemble de cardinal $n-1$. Dans les deux cas, on pose alors $\psi(P)=\psi\left(P^{\prime}\right) \circ \psi(p)$. On a $\phi \psi=$ Id car $\phi$ est un morphisme d'opérades.

Pour montrer que $\psi$ ne dépend pas des choix faits, on fixe deux choix différents et on distingue deux cas. Si les deux choix sont disjoints, au sens où les paires d'éléments choisis sont sans intersection, on utilise l'axiome d'associativité des opérades. Si les deux choix ont un élément en commun, on utilise les relations (19), (20), (21) ou (25).

Les autres relations de Pasc servent à choisir des représentants particuliers des éléments de Quot dans $\mathcal{F}$. Plus précisément, on élimine les compositions du type du côté gauche des relations (22), (23) et (24). Ces représentants peuvent s'écrire comme composition de telle façon que leur image par $\phi$ soit une composition du type de celles utilisées pour définir $\psi$.

Ceci permet de démontrer, en utilisant le fait que $\psi$ ne dépend pas des choix, que $\psi \phi=\mathrm{Id}$, donc $\psi$ est un inverse de $\phi$ pour les parties des ensembles à $n$ éléments, ce qui termine la récurrence.

La différentielle de Pasc est donc uniquement déterminée par le respect de la structure d'opérade et les conditions suivantes :

$$
\begin{aligned}
d\left(e_{1} \wedge e_{2}\right) & =e_{1}-e_{2}, \\
d\left(e_{1}\right) & =0 .
\end{aligned}
$$

On en déduit la description suivante des Pasc-algèbres.

DÉFInition 2. - Une algèbre pascale est la donnée d'un complexe de chaînes $V$, d'applications $\dashv: V \otimes V \rightarrow V$ et $\times: V \otimes V \rightarrow V[-1]$ telles que,

TOME $130-2002-\mathrm{N}^{\mathrm{O}} 2$ 
pour tous $x, y, z$ dans $V$,

$$
\begin{aligned}
x \dashv(y \dashv z) & =(-1)^{y z} x \dashv(z \dashv y)=(x \dashv y) \dashv z, \\
(x \times y) \dashv z & =x \times(y \dashv z), \\
(x \dashv y) \times z & =(-1)^{y z+y} x \times(z \dashv y), \\
x \dashv(y \times z) & =0 \\
x \times y & =(-1)^{x y+x+y+1} y \times x, \\
(x \times y) \times z & =x \times(y \times z)
\end{aligned}
$$

et

$$
\begin{aligned}
(d x \times y)+(-1)^{x+1}(x \times d y)-d(x \times y) & =x \dashv y-(-1)^{x y} y \dashv x, \\
(d x \dashv y)+(-1)^{x}(x \dashv d y)-d(x \dashv y) & =0 .
\end{aligned}
$$

Remarque 3. - On peut penser que Pasc est de Koszul.

On observe que les éléments de dimension nulle de Pasc forment une sousopérade (de différentielle nulle) isomorphe à l'opérade Perm des digèbres commutatives.

D'autre part, on a un morphisme quotient de Pasc dans Com muni de la différentielle nulle. C'est un quasi-isomorphisme car les complexes sous-jacents de Pasc sont les complexes simpliciaux de simplexes, de sorte que l'homologie est en degré 0 avec action triviale des groupes symétriques.

\section{Opérade $K$}

3.1. Algèbres sur associaèdres. - Dans un article antérieur [2], on a défini une opérade différentielle graduée $K$ sur les complexes de cochaînes cellulaires des associaèdres. Cette opérade provient d'une opérade non-symétrique $K^{\prime}$, qui est celle que l'on considère ici. On travaille dans cette section avec des opérades non-symétriques. Tous les résultats énoncés ci-dessous pour des opérades non-symétriques peuvent être traduits en résultats similaires pour les opérades symétriques associées.

3.2. Présentation par générateurs et relations. - On rappelle que $K^{\prime}(n)$ est le complexe de cochaînes cellulaires de l'associaèdre ou polytope de Stasheff de dimension $n-1$ dont les cellules sont en bijection avec les arbres plans à $n+1$ feuilles. On renvoie à [2] pour la définition de la composition.

Proposition 8. - L'opérade $K^{\prime}$ est engendrée par $K^{\prime}(\{1,2\})$. 
Démonstration. - Par récurrence sur $n$. Soit donc $n \geq 3$ et $T$ un arbre dans $K^{\prime}(n)$, la racine en bas. On considère un sommet maximal $s$ de $T$, au sens où aucun sommet ne se trouve greffé au dessus de lui.

Si $s$ est binaire, on choisit de plus un côté, droite ou gauche, et on obtient $T$ comme composé d'un arbre binaire à trois feuilles dans un arbre $T^{\prime}$ à $n$ feuilles obtenu en supprimant $s$.

Si $s$ n'est pas binaire, on choisit de plus une feuille non-extrême de ce sommet, et on obtient $T$ comme composé d'une corolle à trois feuilles dans un arbre $T^{\prime}$ à $n$ feuilles obtenu en supprimant l'arête issue de la feuille non-extrême choisie.

Comme $T^{\prime}$ est composé à partir d'éléments de $K^{\prime}(\{1,2\})$ par hypothèse de récurrence, il en va de même pour $T$. Ceci termine la récurrence.

On a dans $K^{\prime}$ les relations suivantes :

$$
\begin{aligned}
(1>2) \circ_{2}(1<2) & =(1<2) \circ_{1}(1>2), \\
(1>2) \circ_{2}(1>2) & =(1>2) \circ_{1}(1>2)+(1>2) \circ_{1}(1<2), \\
(1<2) \circ_{1}(1<2) & =(1<2) \circ_{2}(1<2)+(1<2) \circ_{2}(1>2), \\
(1>2) \circ_{2}(1 \mid 2) & =(1 \mid 2) \circ_{1}(1>2), \\
(1<2) \circ_{1}(1 \mid 2) & =(1 \mid 2) \circ_{2}(1<2), \\
(1 \mid 2) \circ_{1}(1<2) & =(1 \mid 2) \circ_{2}(1>2), \\
(1 \mid 2) \circ_{1}(1 \mid 2) & =-(1 \mid 2) \circ_{2}(1 \mid 2) .
\end{aligned}
$$

Proposition 9. - L'opérade $K^{\prime}$ est isomorphe au quotient de l'opérade nonsymétrique libre sur $K^{\prime}(\{1,2\})$ par l'idéal engendré par les relations ci-dessus. En particulier $K^{\prime}$ est une opérade quadratique binaire.

Démonstration. - Soit $\mathcal{F}$ l'opérade libre sur $K^{\prime}(\{1,2\})$, Rel les relations ci-dessus et Quot l'opérade quotient de $\mathcal{F}$ par l'idéal engendré par Rel. Par la proposition 8, on a un morphisme surjectif d'opérades $\phi$ de Quot $\rightarrow K$. La démonstration consiste à construire un inverse $\psi$ par récurrence.

On a un inverse pour les arbres à 2 feuilles. Supposons donc un inverse $\psi$ construit jusqu'aux arbres à $n$ feuilles. Soit $T$ un arbre à $n+1$ feuilles. Pour définir $\psi(T)$, on choisit un sommet maximal $s$, comme dans la démonstration de la proposition 8, et on obtient $T$ comme composé d'un arbre $t$ à trois feuilles dans un arbre $T^{\prime}$ à $n$ feuilles. On pose alors $\psi(T)=\psi\left(T^{\prime}\right) \circ \psi(t)$. Comme $\phi$ est un morphisme d'opérades, on a $\phi \psi=\mathrm{Id}$.

On montre que $\psi$ ne dépend pas des choix faits. On appelle secteur d'un arbre plan une paire de feuilles consécutives. Chaque choix possible pour obtenir $T$ par composition correspond à une paire de secteurs consécutifs. Considérons deux choix distincts. Si les paires de secteurs sont disjointes, l'axiome

TOME $130-2002-\mathrm{N}^{\mathrm{O}} 2$ 
d'associativité des opérades montre l'indépendance voulue. Sinon, on utilise les relations (36), (41) ou (42) de $K^{\prime}$.

Les autres relations de $K^{\prime}$ servent à choisir des représentants particuliers des éléments de Quot dans $\mathcal{F}$. Plus précisément, on supprime les compositions de la forme du côté gauche de (37), (38), (39) et (40). Les représentants obtenus peuvent s'écrire comme composition de telle façon que leur image par $\phi$ soit une composition du type de celles utilisées pour définir $\psi$.

Ceci permet de démontrer, en utilisant le fait que $\psi$ ne dépend pas des choix, que $\psi \phi=\mathrm{Id}$, donc $\psi$ est un inverse de $\phi$ pour les arbres à $n+1$ feuilles, ce qui termine la récurrence.

La différentielle de $K^{\prime}$ est donc uniquement déterminée par le respect de la structure d'opérade et les conditions suivantes :

$$
\begin{aligned}
& d(1<2)=-d(1>2)=(1 \mid 2), \\
& d(1 \mid 2)=0 .
\end{aligned}
$$

On en déduit la description suivante des $K^{\prime}$-algèbres, qui est aussi celle des $K$-algèbres.

DÉfinition 3. - Une $K$-algèbre est la donnée d'un complexe de cochaînes $V$ et d'applications $\succ: V \otimes V \rightarrow V, \prec: V \otimes V \rightarrow V$ et $\times: V \otimes V \rightarrow V[-1]$ telles que pour tous $x, y, z$ dans $V$,

$$
\begin{aligned}
x \succ(y \prec z) & =(x \succ y) \prec z, \\
x \succ(y \succ z) & =(x \succ y) \succ z+(x \prec y) \succ z, \\
(x \prec y) \prec z & =x \prec(y \prec z)+x \prec(y \succ z), \\
(x \succ y) \times z & =x \succ(y \times z), \\
x \times(y \prec z) & =(x \times y) \prec z, \\
v(x \prec y) \times z & =(-1)^{y} x \times(y \succ z), \\
(x \times y) \times z & =x \times(y \times z)
\end{aligned}
$$

et

$$
\begin{aligned}
(d x \prec y)+(-1)^{x}(x \prec d y)-d(x \prec y) & =(-1)^{x} x \times y, \\
(d x \succ y)+(-1)^{x}(x \succ d y)-d(x \succ y) & =(-1)^{x+1} x \times y, \\
(d x \times y)+(-1)^{x+1}(x \times d y)-d(x \times y) & =0 .
\end{aligned}
$$

REMARQUe 4. - La koszulité d'une version non-graduée de l'opérade $K^{\prime}$ est annoncée dans [9]. On peut penser que $K^{\prime}$ est également de Koszul.

BULletin DE LA SOCiÉtÉ MATHÉmATiQUe DE FRANCE 
On observe que le quotient de $K^{\prime}$ par les éléments de dimension non nulle forme une opérade (de différentielle nulle) isomorphe à l'opérade Dend des algèbres dendriformes.

D'autre part, on a un morphisme de As muni de la différentielle nulle dans $K^{\prime}$. C'est un quasi-isomorphisme, car les complexes sous-jacents à $K^{\prime}$ sont les complexes cellulaires des polytopes de Stasheff.

\section{Opérades duales}

On donne ici une présentation par générateurs et relations des opérades duales des trois opérades précédentes. On démontre aussi que l'opérade (nonsymétrique) duale de $K^{\prime}$ est isomorphe à l'opérade Pasc vue comme opérade non-symétrique.

On obtient les relations du dual d'une opérade quadratique binaire par le calcul de l'orthogonal des relations de cette opérade. Plus précisément, pour une opérade engendrée par $E=E(2)$, on considère, dans l'opérade libre $\mathcal{F}_{E}$ engendrée par $E$, le sous- $\mathfrak{S}_{3}$-module $R$ de $\mathcal{F}_{E}(3)$ engendré par les relations. On identifie $\mathcal{F}_{E}(3)$ avec

$$
E \otimes E \oplus \tau E \otimes E \oplus \tau^{2} E \otimes E
$$

par l'application

$$
\tau^{k}(x \otimes y) \longmapsto \tau^{k}\left(x \circ_{1} y\right)
$$

Soit $E^{\prime}$ le dual de $E$, tensorisé par la représentation signe de $\mathfrak{S}_{2}$. On définit un couplage entre $\mathcal{F}_{E}(3)$ et $\mathcal{F}_{E^{\prime}}(3)$ en prenant la somme directe de trois couplages entre $E \otimes E$ et $E^{\prime} \otimes E^{\prime}$ pour chacune des puissances de $\tau$. On prend alors l'orthogonal de $R$ pour ce couplage. De même, la différentielle de l'opérade duale est obtenue par transposition. On renvoie à [4] pour les fondations théoriques de cette dualité quadratique.

4.1. Trigèbres. - On travaille dans ce paragraphe avec des opérades nonsymétriques. L'opérade Trias' est la duale quadratique de l'opérade $K^{\prime}$ des associaèdres. On note Trias l'opérade symétrique associée.

TOME $130-2002-\mathrm{N}^{\mathrm{O}} 2$ 
L'opérade Trias' est engendrée par les éléments $1 \dashv 2,1 \vdash 2$ de dimension 0 et $1 \times 2$ de dimension 1 soumis aux relations suivantes:

$$
\begin{aligned}
& (1 \dashv 2) \circ_{1}(1 \vdash 2)=(1 \vdash 2) \circ_{2}(1 \dashv 2), \\
& (1 \dashv 2) \circ_{2}(1 \dashv 2)=(1 \dashv 2) \circ_{2}(1 \vdash 2), \\
& (1 \dashv 2) \circ_{1}(1 \dashv 2)=(1 \dashv 2) \circ_{2}(1 \vdash 2), \\
& (1 \vdash 2) \circ_{1}(1 \vdash 2)=(1 \vdash 2) \circ_{1}(1 \dashv 2), \\
& (1 \vdash 2) \circ_{2}(1 \vdash 2)=(1 \vdash 2) \circ_{1}(1 \dashv 2), \\
& (1 \times 2) \circ_{1}(1 \vdash 2)=(1 \vdash 2) \circ_{2}(1 \times 2), \\
& (1 \times 2) \circ_{2}(1 \dashv 2)=(1 \dashv 2) \circ_{1}(1 \times 2), \\
& (1 \times 2) \circ_{1}(1 \dashv 2)=(1 \times 2) \circ_{2}(1 \vdash 2), \\
& (1 \vdash 2) \circ_{1}(1 \times 2)=0, \\
& (1 \dashv 2) \circ_{2}(1 \times 2)=0, \\
& (1 \times 2) \circ_{1}(1 \times 2)=-(1 \times 2) \circ_{2}(1 \times 2) .
\end{aligned}
$$

La différentielle est uniquement déterminée par le respect de la structure d'opérade et les conditions suivantes :

$$
\begin{aligned}
& d(1 \times 2)=1 \dashv 2-1 \vdash 2, \\
& d(1 \vdash 2)=d(1 \dashv 2)=0 .
\end{aligned}
$$

On en déduit la description suivante des trigèbres.

DÉFInition 4. - Une trigèbre est la donnée d'un complexe de chaines $V$, d'applications $\vdash: V \otimes V \rightarrow V, \dashv: V \otimes V \rightarrow V$ et $\times: V \otimes V \rightarrow V[-1]$ telles que

$$
\begin{aligned}
(x \vdash y) \dashv z & =x \vdash(y \dashv z), \\
x \dashv(y \dashv z) & =x \dashv(y \vdash z)=(x \dashv y) \dashv z, \\
(x \vdash y) \vdash z & =(x \dashv y) \vdash z=x \vdash(y \vdash z), \\
(x \vdash y) \times z & =x \vdash(y \times z), \\
(x \times y) \dashv z & =x \times(y \dashv z), \\
v(x \dashv y) \times z & =(-1)^{y} x \times(y \vdash z), \\
(x \times y) \vdash z & =0, \\
x \dashv(y \times z) & =0, \\
(x \times y) \times z & =x \times(y \times z)
\end{aligned}
$$

BULLETIN DE LA SOCiÉtÉ MATHÉMATIQUE DE FRANCE 
et

$$
\begin{aligned}
(d x \times y)+(-1)^{x+1}(x \times d y)-d(x \times y) & =x \dashv y-x \vdash y, \\
(d x \vdash y)+(-1)^{x}(x \vdash d y)-d(x \vdash y) & =0, \\
(d x \dashv y)+(-1)^{x}(x \dashv d y)-d(x \dashv y) & =0 .
\end{aligned}
$$

On peut donner une description globale de Trias'. Si $\mathcal{P}$ est une opérade symétrique, on note $\mathcal{P}_{\sharp \downarrow}$ l'opérade non symétrique obtenue par oubli des actions des groupes symétriques.

Proposition 10. - L'opérade non-symétrique Trias' est isomorphe à l'opérade non-symétrique $\mathrm{Pasc}_{\mathbb{Z}}$.

Démonstration. - On change de notation : les éléments $e_{1}$, e $e_{2}$ et $e_{1} \wedge e_{2}$ de Pasc deviennent les générateurs $1 \dashv 2,1 \vdash 2$ et $1 \times 2$ de Trias'. On vérifie sans difficulté que les relations qui définissent Trias sont vérifiées dans $\mathrm{Pasc}_{\not{Z}}$. Il reste à démontrer que l'on obtient bien ainsi une présentation par générateurs et relations de $\mathrm{Pasc}_{\not{Z}}$. On voit aisément que les trois éléments $1 \dashv 2,1 \vdash 2$ et $1 \times 2$ engendrent $\mathrm{Pasc}_{\mathbb{Z}}$. Il reste à démontrer que les relations de Trias' sont exactement celles de $\mathrm{Pasc}_{\not{Z}}$. On ne détaille pas la preuve, qui est assez semblable à celle de la proposition 7 .

Remarque 5. - En particulier, on obtient ainsi la série génératrice de Trias.

On observe que la sous-opérade (de différentielle nulle) formée par les éléments de dimension nulle de Trias est isomorphe à l'opérade Dias des digèbres.

D'autre part, on a un morphisme de Trias dans As muni de la différentielle nulle. C'est un quasi-isomorphisme car il provient d'un morphisme nonsymétrique de Trias' dans As $^{\prime}$ qui s'identifie au quasi-isomorphisme de Pasc dans Com.

Si $\mathcal{P}$ est une opérade de $\mathbb{Z}$-modules, on note $\mathcal{P}_{\mathbb{Q}}$ l'opérade d'espace vectoriels sur $\mathbb{Q}$ obtenue par extension des scalaires.

Proposition 11. - On a un isomorphisme d'opérades symétriques

$$
(\operatorname{Pasc} \otimes \mathrm{As})_{\mathbb{Q}} \simeq \operatorname{Trias}_{\mathbb{Q}}
$$

Démonstration. - On vérifie que les relations de Trias sont celles de l'opérade Pasc $\circ$ As où le produit $\circ$ est le produit de Manin des opérades quadratiques binaires, voir [4, 2.2]. Par conséquent, Trias est la sous-opérade de Pasc $\otimes$ As engendrée par $\operatorname{Pasc}(2) \otimes \operatorname{As}(2)$. Comme on a égalité des séries génératrices, on peut en déduire un isomorphisme des opérades tensorisées par $\mathbb{Q}$.

TOME $130-2002-\mathrm{N}^{\mathrm{O}} 2$ 
4.2. Algèbres $\amalg$. — Cette opérade est la duale quadratique de l'opérade $\Pi$ sur les permutoèdres. Elle est engendrée par les éléments suivants : [1,2] symétrique de dimension $1,\langle 1,2\rangle$ et $\langle 2,1\rangle$ de dimension 0 , modulo l'idéal engendré par les relations suivantes :

$$
\begin{aligned}
\langle 1,2\rangle \circ_{1}\langle 1,2\rangle-\tau\left(\langle 1,2\rangle \circ_{1}\langle 2,1\rangle\right) & +\tau^{2}\left(\langle 2,1\rangle \circ_{1}\langle 2,1\rangle\right), \\
\tau^{2}\left([1,2] \circ_{1}\langle 1,2\rangle\right)+\tau\left([1,2] \circ_{1}\langle 2,1\rangle\right) & =\langle 1,2\rangle \circ_{1}[1,2], \\
\langle 2,1\rangle \circ_{1}[1,2] & =0, \\
{[1,2] \circ_{1}[1,2]+\tau\left([1,2] \circ_{1}[1,2]\right) } & +\tau^{2}\left([1,2] \circ_{1}[1,2]\right)=0,
\end{aligned}
$$

où $\tau$ est le cycle $3 \rightarrow 2 \rightarrow 1 \rightarrow 3$. La différentielle est uniquement déterminée par le respect de la structure d'opérade et les conditions suivantes :

$$
\begin{aligned}
d([1,2]) & =\langle 1,2\rangle+\langle 2,1\rangle, \\
d(\langle 1,2\rangle) & =0 .
\end{aligned}
$$

On en déduit la description suivante des $\amalg$-algèbres.

DÉfinition 5. - Une Ш-algèbre est la donnée d'un complexe de chaînes $V$, d'applications $\langle\cdot, \cdot\rangle: V \otimes V \rightarrow V$ et $[\cdot, \cdot]: V \otimes V \rightarrow V[-1]$ telles que

$$
\begin{gathered}
\langle x,\langle y, z\rangle\rangle=\langle\langle x, y\rangle, z\rangle-\langle\langle x, z\rangle, y\rangle, \\
\langle[x, y], z\rangle=(-1)^{y z+z}[\langle x, z\rangle, y]+[x,\langle y, z\rangle], \\
\langle x,[y, z]\rangle=0, \\
{[x, y]=(-1)^{x y+x+y}[y, x],} \\
(-1)^{y+z x}[[x, y], z]+(-1)^{z+x y}[[y, z], x]+(-1)^{x+y z}[[z, x], y]=0
\end{gathered}
$$

et

$$
\begin{aligned}
& {[d x, y]+(-1)^{x+1}[x, d y]-d([x, y]) }=\langle x, y\rangle+(-1)^{x y}\langle y, x\rangle, \\
&(\langle d x, y\rangle)+(-1)^{x}(\langle x, d y\rangle-d(\langle x, y\rangle)=0 .
\end{aligned}
$$

La sous-opérade (de différentielle nulle) formée par les éléments de dimension nulle de $\amalg$ est isomorphe à l'opérade Leib des algèbres de Leibniz.

D'autre part, on a un morphisme de $\amalg$ dans Lie muni de la différentielle nulle. C'est un quasi-isomorphisme, comme application duale du quasi-isomorphisme de Com dans $\Pi$, car la construction Bar préserve les quasi-isomorphismes.

Proposition 12. - On a un isomorphisme $(\operatorname{Pasc} \otimes \mathrm{Lie})_{\mathbb{Q}} \simeq \amalg_{\mathbb{Q}}$.

Démonstration. - On vérifie que les relations de $\amalg$ sont celles de l'opérade Pasc $\circ$ Lie où le produit $\circ$ est le produit de Manin des opérades quadratiques BULletin DE LA SOCiÉtÉ MATHÉmATiQUe DE FRANCE 
binaires, voir $[4,2.2]$. Par conséquent, $\amalg$ est la sous-opérade de Pasc $\otimes$ Lie engendrée par $\operatorname{Pasc}(2) \otimes \operatorname{Lie}(2)$. Comme on a égalité des séries génératrices, on peut en déduire un isomorphisme des opérades tensorisées par $\mathbb{Q}$.

4.3. Algèbres $\boldsymbol{\Lambda}$. - Cette opérade est la duale quadratique de l'opérade Pasc sur les simplexes. Elle a une saveur « espace de configurations », voir par exemple sa série caractéristique supposée dans le tableau récapitulatif. Elle est engendrée par les éléments suivants : [1,2] symétrique de dimension $1,1 \curvearrowleft 2$ et $2 \curvearrowleft 1$ de dimension 0 , modulo l'idéal engendré par les relations suivantes :

$$
\begin{aligned}
\tau^{2}\left((2 \curvearrowleft 1) \circ_{1}(1 \curvearrowleft 2)\right)-(1 \curvearrowleft 2) \circ_{1}(1 \curvearrowleft 2) \\
\quad=\tau^{2}\left((2 \curvearrowleft 1) \circ_{1}(2 \curvearrowleft 1)\right)-\tau\left((1 \curvearrowleft 2) \circ_{1}(2 \curvearrowleft 1)\right),
\end{aligned}
$$

$$
\begin{array}{r}
{[1,2] \circ_{1}(1 \curvearrowleft 2)-\tau^{2}\left([1,2] \circ_{1}(2 \curvearrowleft 1)\right)=\tau\left((1 \curvearrowleft 2) \circ_{1}[1,2]\right),} \\
{[1,2] \circ_{1}[1,2]+\tau\left([1,2] \circ_{1}[1,2]\right)+\tau^{2}\left([1,2] \circ_{1}[1,2]\right)=0,}
\end{array}
$$

où $\tau$ est le cycle $3 \rightarrow 2 \rightarrow 1 \rightarrow 3$. La différentielle est uniquement déterminée par le respect de la structure d'opérade et les conditions suivantes :

$$
\begin{gathered}
d(1 \curvearrowleft 2)=[1,2], \\
d([1,2])=0 .
\end{gathered}
$$

On en déduit la description suivante des $\Lambda$-algèbres.

DÉFinition 6. - Une $\Lambda$-algèbre est la donnée d'un complexe de cochaînes $V$, d'applications $\curvearrowleft: V \otimes V \rightarrow V$ et $[\cdot, \cdot]: V \otimes V \rightarrow V[-1]$ telles que

$$
\begin{gathered}
(x \curvearrowleft y) \curvearrowleft z-x \curvearrowleft(y \curvearrowleft z)=(x \curvearrowleft z) \curvearrowleft y-x \curvearrowleft(z \curvearrowleft y), \\
{[x, y] \curvearrowleft z=(-1)^{z y+z}[x \curvearrowleft z, y]+[x, y \curvearrowleft z],} \\
{[x, y]=(-1)^{x y+x+y}[y, x],} \\
(-1)^{y+z x}[[x, y], z]+(-1)^{z+x y}[[y, z], x]+(-1)^{x+y z}[[z, x], y]=0
\end{gathered}
$$

et

$$
\begin{gathered}
(d x \curvearrowleft y)+(-1)^{x}(x \curvearrowleft d y)-d(x \curvearrowleft y)=(-1)^{x}[x, y], \\
([d x, y])+(-1)^{x+1}[x, d y]-d([x, y])=0 .
\end{gathered}
$$

On observe que le quotient de $\Lambda$ par les éléments de dimension non nulle forme une opérade (de différentielle nulle) isomorphe à l'opérade PreLie des algèbres pré-Lie.

D'autre part, on a un morphisme de l'opérade Lie (munie de la différentielle nulle) dans $\Lambda$.

TOME $130-2002-\mathrm{N}^{\mathrm{O}} 2$ 


\section{Morphismes horizontaux}

Il reste, pour compléter la description du diagramme (1), à décrire les applications horizontales des lignes 2 et 4 . Les autres morphismes horizontaux ont déjà été définis, voir par exemple [8] ; on peut les retrouver par restriction ou passage au quotient de ceux qu'on définit ci-dessous. On considère ici $K$ et Trias comme des opérades avec action libre des groupes symétriques.

On a un morphisme d'opérades de $K$ dans $\Pi$ défini au niveau des algèbres par les formules suivantes :

$$
\begin{aligned}
& x \prec y:=x \prec y, \\
& x \succ y:=(-1)^{x y} y \prec x, \\
& x \times y:=x \times y .
\end{aligned}
$$

Le morphisme d'opérades de $\Lambda$ dans $K$ est défini au niveau des algèbres par les formules suivantes :

$$
\begin{aligned}
x \curvearrowleft y & :=x \prec y-(-1)^{x y} y \succ x, \\
{[x, y] } & :=x \times y+(-1)^{x y+x+y} y \times x .
\end{aligned}
$$

Les formules suivantes définissent, au niveau des algèbres, un morphisme d'opérades de Trias dans Pasc :

$$
\begin{aligned}
& x \dashv y:=x \dashv y, \\
& x \vdash y:=(-1)^{x y} y \dashv x, \\
& x \times y:=x \times y .
\end{aligned}
$$

On a un morphisme d'opérades de $\amalg$ dans Trias défini au niveau des algèbres par les formules suivantes :

$$
\begin{aligned}
\langle x, y\rangle & :=x \dashv y-(-1)^{x y} y \vdash x, \\
{[x, y] } & :=x \times y+(-1)^{x y+x+y} y \times x .
\end{aligned}
$$

On vérifie alors sans difficulté que

Proposition 13. - Le diagramme (1) est formé de carrés commutatifs.

De plus, chacune des lignes de ce diagramme est « exacte», au sens suivant : l'opérade de gauche est le quotient de l'opérade du milieu par l'idéal engendré par l'image de l'idéal d'augmentation de l'opérade de droite.

BULLETIN DE LA SOCiÉtÉ MATHÉMATIQUE DE FRANCE 


\section{Séries génératrices}

On utilise la convention suivante pour les séries génératrices d'opérades différentielles graduées:

$$
g_{\mathcal{P}}(x, t)=\sum_{n \geq 1} \sum_{k} \operatorname{dim} \mathcal{P}^{k}(n)(-t)^{k} \frac{x^{n}}{n !} .
$$

La série génératrice de la suspension $\Sigma \mathcal{P}$ d'une opérade $\mathcal{P}$ vérifie

$$
g_{\Sigma \mathcal{P}}(x, t)=-g_{\mathcal{P}}(-t x, t) / t .
$$

Les séries génératrices des opérades qui apparaissent dans le diagramme (1) sont récapitulées dans le tableau 1. Celles des opérades des première, troisième et cinquième lignes sont bien connues. Celles des opérades Pasc, $K, \Pi$ et Trias s'obtiennent aisément en partant de la description explicite de ces opérades. Celle de $\amalg$ résulte de la koszulité de $\Pi$. Enfin, celle de $\Lambda$ s'obtient par inversion de celle de Pasc, sous réserve que les opérades Pasc et $\Lambda$ soient de Koszul.

\section{BIBLIOGRAPHIE}

[1] Chapoton (F.) - Un endofoncteur de la catégorie des opérades, in Dialgebras and related operads, Lecture Notes in Math., vol. 1763, Springer, 2001, pp. 105-110.

[2] _ Construction de certaines opérades et bigèbres associées aux polytopes de Stasheff et hypercubes, Trans. Amer. Math. Soc., t. 354 (2002), no. 1, pp. 63-74.

[3] Chapoton (F.) \& Livernet (M.) - Pre-Lie algebras and the rooted trees operad, Internat. Math. Res. Notices, t. 8 (2001), pp. 395-408.

[4] Ginzburg (V.) \& Kapranov (M.) - Koszul duality for operads, Duke Math. J., t. 76 (1994), no. 1, pp. 203-272.

[5] LODAY (J.-L.) - Une version non commutative des algèbres de Lie : les algèbres de Leibniz, Enseign. Math., t. 39 (1993), pp. 269-293.

[6] _ Algèbres ayant deux opérations associatives (digèbres), C. R. Acad. Sci. Paris, Sér. I Math., t. 321 (1995), pp. 141-146.

[7] _ Overview on Leibniz algebras, dialgebras and their homology, in Cyclic cohomology and noncommutative geometry (Waterloo, ON, 1995), Amer. Math. Soc., Providence, RI, 1997, pp. 91-102.

[8] , Dialgebras, in Dialgebras and related operads, Lecture Notes in Math., vol. 1763, Springer, 2001, pp. 7-66.

[9] Loday (J.-L.) \& Ronco (M.O.) - Une dualité entre simplexes standards et polytopes de Stasheff, C. R. Acad. Sci. Paris, Sér. I Math., t. 333 (2001), pp. 81-86.

[10] Markl (M.) - Distributive laws and Koszulness, Ann. Inst. Fourier (Grenoble), t. 46 (1996), no. 2, pp. 307-323.

TOME $130-2002-\mathrm{N}^{\mathrm{O}} 2$ 


\begin{tabular}{|c|c|c|}
\hline $\begin{array}{c}\text { Zin : } \\
\frac{x}{1-x} \\
\sum n ! \frac{x^{n}}{n !}\end{array}$ & $\begin{array}{c}\text { Dend : } \\
\frac{(1-2 x)-\sqrt{(1-4 x)}}{2 x} \\
\sum \frac{1}{n+1}\left(\begin{array}{c}2 n \\
n\end{array}\right) x^{n}\end{array}$ & $\sum n^{n-1} \frac{x^{n}}{n !}$ \\
\hline $\begin{array}{c}\Pi: \\
\frac{\mathrm{e}^{t x}-1}{1+(t-1) \mathrm{e}^{t x}} \\
\sum \pi_{n, k}(-t)^{k} \frac{x^{n}}{n !}\end{array}$ & $\begin{array}{c}K: \\
\frac{(1-2 x+x t)}{2 x(1-t)} \\
-\frac{\sqrt{1-4 x+2 x t+x^{2} t^{2}}}{2 x(1-t)} \\
\sum c_{n, k}(-t)^{k} x^{n}\end{array}$ & $\sum \prod_{k=1}^{n-1}(n-k t) \frac{x^{n}}{n !}$ \\
\hline $\begin{array}{l}\text { Com : } \\
\mathrm{e}^{x}-1 \\
\sum \frac{x^{n}}{n !}\end{array}$ & $\begin{array}{l}\text { As : } \\
\frac{x}{1-x} \\
\sum x^{n}\end{array}$ & $\begin{array}{c}\text { Lie : } \\
-\ln (1-x) \\
\sum(n-1) ! \frac{x^{n}}{n !}\end{array}$ \\
\hline $\begin{array}{c}\text { Pasc : } \\
\frac{\mathrm{e}^{x}-\mathrm{e}^{(1-t) x}}{t} \\
\sum\left(\begin{array}{c}n \\
k+1\end{array}\right)(-t)^{k} \frac{x^{n}}{n !}\end{array}$ & $\begin{array}{c}\text { Trias : } \\
\frac{1}{t}\left(\frac{x}{1-x}-\frac{(1-t) x}{1+(t-1) x}\right) \\
\sum\left(\begin{array}{c}n \\
k+1\end{array}\right)(-t)^{k} x^{n}\end{array}$ & $\begin{array}{c}\amalg: \\
-\frac{\ln (1-x)}{t}+\frac{\ln (1+(t-1) x)}{t} \\
\sum\left(\begin{array}{c}n \\
k+1\end{array}\right)(n-1) !(-t)^{k} \frac{x^{n}}{n !}\end{array}$ \\
\hline $\begin{array}{c}\text { Perm : } \\
x \mathrm{e}^{x} \\
\sum n \frac{x^{n}}{n !}\end{array}$ & $\begin{array}{l}\text { Dias : } \\
\frac{x}{(1-x)^{2}} \\
\sum n x^{n}\end{array}$ & $\begin{array}{c}\text { Leib : } \\
\frac{x}{1-x} \\
\sum n ! \frac{x^{n}}{n !}\end{array}$ \\
\hline
\end{tabular}

TABEAU 1. Dans ce tableau, $\pi_{n, k}\left(\right.$ resp. $\left.c_{n, k}\right)$ désigne le nombre de faces de dimension $k$ du permutoèdre (resp. de l'associaèdre) de dimension $n-1$. 